\title{
Predicting the effects of urban development on land transition and spatial patterns of land use in western Peninsular Malaysia
}

\begin{abstract}
Analyzing the effects of urban development on dynamic and spatial patterns of land use is vital to establish more efficient land management policies. However, in Malaysia, such effects are usually explained without quantitative metrics. This research quantified the future impact of urban expansion on the dynamic of land use by developing the area-independent dynamic metric. The metric was calculated based on summarizing the cross tabulation matrices of change in an urbanizing area at west coast of Peninsular Malaysia. Another two land use measures involving vulnerability to gain and vulnerability to loss were used to evaluate tendency of land classes to transition. The effects of urban development on spatial patterns of land use were quantified using two landscape metrics involving the Edge Density (ED) and Area-Weighted Mean Patch Fractal Dimension (AWMPFD). Analyses were carried out on a set of spatial land use data including observed 1997, 2002, and 2008, as well as a simulated near future land change for the year 2020 under a spatio-temporal land use model. Results showed that urban development practices would influence the dynamic of land transition in the near future. Urban growth would experience a fast-growing dynamic and high vulnerability to gain than loss while the dynamic and vulnerability of forest/wetland covers would decrease in terms of loss. Moreover, agriculture practices tend to be hindered by further urban development in the coming years. Another important finding was that urban development process would influence the spatial patterns of land use in the near future.
\end{abstract}

Keyword: Area-independent dynamic; Land transition; Landscape metrics; Pattern; Policy; Vulnerability 